University of Rhode Island

DigitalCommons@URI

Mechanical, Industrial \& Systems Engineering

Faculty Publications

Mechanical, Industrial \& Systems Engineering

3-27-2015

\title{
Systematic Approach to Using Isentropic Stress Reverberation Techniques in Approximating Equation of State
}

\author{
Gifford Plume \\ Carl-Ernst Rousseau \\ University of Rhode Island, roussce@uri.edu
}

Follow this and additional works at: https://digitalcommons.uri.edu/mcise_facpubs

Terms of Use

All rights reserved under copyright.

\section{Citation/Publisher Attribution}

Plume G., \& Rousseau C.E. (2015). Systematic approach to using isentropic stress reverberation techniques in approximating equation of state. Review of Scientific Instruments. 86: 033908. doi: 10.1063/1.4914023

Available at: http://dx.doi.org/10.1063/1.4914023

This Article is brought to you for free and open access by the Mechanical, Industrial \& Systems Engineering at DigitalCommons@URI. It has been accepted for inclusion in Mechanical, Industrial \& Systems Engineering Faculty Publications by an authorized administrator of DigitalCommons@URI. For more information, please contact digitalcommons-group@uri.edu. 


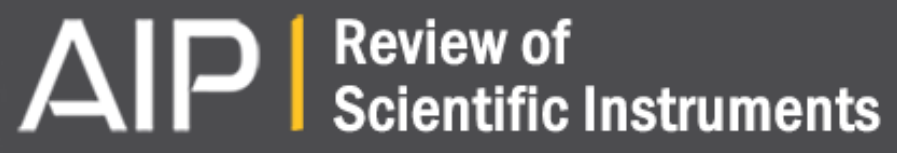

\section{Systematic approach to using isentropic stress reverberation techniques in approximating equation of state}

Gifford Plume and Carl-Ernst Rousseau

Citation: Review of Scientific Instruments 86, 033908 (2015); doi: 10.1063/1.4914023

View online: http://dx.doi.org/10.1063/1.4914023

View Table of Contents: http://scitation.aip.org/content/aip/journal/rsi/86/3?ver=pdfcov

Published by the AIP Publishing

\section{Articles you may be interested in}

Examining the temporal evolution of hypervelocity impact phenomena via high-speed imaging and ultravioletvisible emission spectroscopy

J. Appl. Phys. 116, 034901 (2014); 10.1063/1.4890230

Hydrodynamic simulation of converging shock waves in porous conical samples enclosed within solid targets

J. Appl. Phys. 110, 053501 (2011); 10.1063/1.3605556

Shock equation of state of multi-constituent epoxy-metal particulate composites

J. Appl. Phys. 109, 013531 (2011); 10.1063/1.3531579

GEKKO/HIPER-driven shock waves and equation-of-state measurements at ultrahigh pressures Phys. Plasmas 11, 1600 (2004); 10.1063/1.1650845

Near-absolute Hugoniot measurements in aluminum to $500 \mathrm{GPa}$ using a magnetically accelerated flyer plate technique

J. Appl. Phys. 94, 4420 (2003); 10.1063/1.1604967

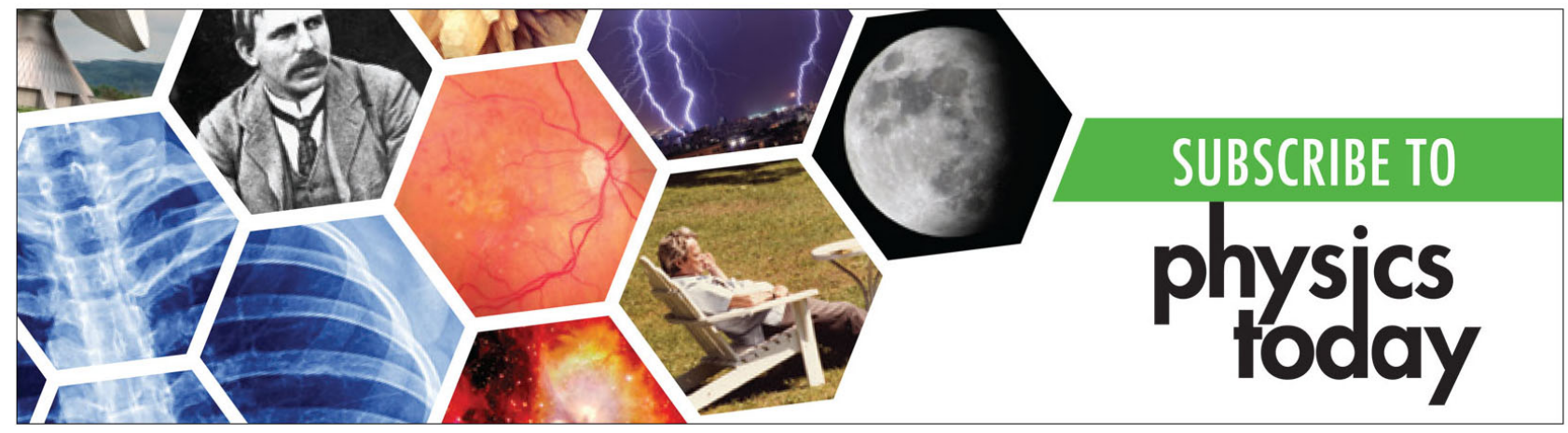




\title{
Systematic approach to using isentropic stress reverberation techniques in approximating equation of state
}

\author{
Gifford Plume and Carl-Ernst Rousseau ${ }^{\mathrm{a})}$ \\ Mechanical Engineering, University of Rhode Island, 92 Upper College Rd., Kingston, \\ Rhode Island 02881, USA
}

(Received 4 January 2015; accepted 16 February 2015; published online 27 March 2015)

\begin{abstract}
Isentropic stress reverberations are used to obtain multiple Hugoniot states from a single plate impact experiment using a layered plate geometry, where a low impedance inner layer is embedded within a high impedance bulk structure. The mathematical framework used in this technique uses the classical Rankine-Hugoniot equations in the method of impedance matching, where the bulk material is required to have a known Hugoniot. Factors including the wave velocities in the materials, input pulse duration, inner layer thickness, and diameter of the test samples affect the number of states that can be generated from a single experiment. Experiments using 6061 aluminum and polycarbonate, respectively, as the bulk material and inner layer, accurately generated six Hugoniot states for the polycarbonate. Experiments using A572 grade 50 structural steel as the bulk material accurately generated ten Hugoniot states for the polycarbonate. For each experiment, the method can be used to generate a Hugoniot equation defining the material response of the inner layer within the domain encompassed by the specific test. The method is also confined to the low to moderate stress regions, within which Hugoniot and isentropic representations of the material are almost identical. (C) 2015 AIP Publishing LLC. [http://dx.doi.org/10.1063/1.4914023]
\end{abstract}

\section{INTRODUCTION}

The formulation of a material's Hugoniot is a recurring theme in literature. ${ }^{1-7}$ When coupled with a valid thermodynamic Equation of State (EOS), it allows the investigator to accurately develop models that predict all states of matter associated with dynamic loading events. Researchers may generate a single Hugoniot state through the capture of two shock properties from an experiment. In cases where a Velocity Interferometer System for Any Reflector (VISAR) is used at a specimen's free surface, the particle velocity information is complemented by measurements of shock velocity through the use of shorting pins, where the transit time through the sample's thickness can be related to the lag time between the activation of the pins and the arrival of the particle velocity signal at the free surface. ${ }^{3,8}$ In cases where stresses are measured with a single embedded stress gage, researchers use the method of impedance matching to obtain particle velocity from measurements of impact velocity. ${ }^{2,4,5,8}$ In these cases, the experiments are designed to be symmetric, with impactor and specimen made of the same material, thus resulting in particle velocities that are half of the impact velocity. Alternatively, where symmetry not to be achievable, an impactor material with a well-defined Hugoniot must be chosen. Researchers have also used several stress gages at fixed locations within a sample to complement the stress measurements with calculated shock velocities that are determined by relating the lag time between the arrival of the shock wave at the various gage locations to the distance between the gages..$^{8-10}$

\footnotetext{
a)rousseau@uri.edu
}

Traditionally, the locus of points necessary to construct a complete Hugoniot is obtained through a rigorous series of experiments, where each test produces a single data point. Nevertheless, for experiments properly designed, a single experiment may occasionally elicit numerous data points. This, however, is strictly limited to low stress regimes.

The use of stress reverberation experiments as a means of extracting multiple data points along the equation of state of a material was introduced by Lysne et al., ${ }^{11}$ using quartz gages. The feasibility of the technique was again reinforced by Rosenberg ${ }^{12}$ who further extended the experimental diagnosis to the use of manganin gages. In both of the above instances, impact was used as a method of stress generation, with the resulting stresses limited to ranges below $10 \mathrm{GPa}$. In those experiments, isentropic results for epoxy and polyurethane coincided with previously established Hugoniot data for these materials.

These nearly perfect correlations are to be expected. Indeed, even though the Hugoniot and the compression isentropes access different areas of the equation of state, they share a common origin, and only diverge significantly at higher values of stress. In a work by Reisman et al., the authors noted that inside an envelope with limits below 50 $\mathrm{GPa}$, Hugoniot and compression isentropes lie within $2 \%$ to $3 \%$ from each other. ${ }^{13}$ The conditions associated with the latter case bring out the advantage of being able to assume complete process reversibility wherein multiple data points can be obtained along the loading path. For the highly compressive state, however, where the gradual loading does steepen into a shock, only a single, final data point can be achieved. Thus, both methods present their respective limitations.

In the present work, an approach similar to that of Rosenberg is followed, with the upper bounds of the pressure 
experienced by the specimens restricted to $10 \mathrm{GPa}$. Yet, within that threshold, the material does experience a transition to plastic flow that can be identified in the stress history by an ephemeral horizontal plateau known as the Hugoniot Elastic Limit (HEL).

Through the entire range described by the equation of state, the Rankine-Hugoniot equations set limits on the physical behavior of the material by imposing laws pertaining to conservation of mass, momentum, and energy ${ }^{14}$

$$
\begin{gathered}
V=V_{0} \frac{U_{s}-\left(u_{p}-u_{0}\right)}{U_{s}}, \\
\sigma=\sigma_{0}+\rho_{0} U_{s}\left(u_{p}-u_{0}\right), \\
\mathrm{E}=\mathrm{E}_{0} \frac{1}{2}\left(\sigma+\sigma_{0}\right)\left(V_{0}-V\right),
\end{gathered}
$$

where $V, \sigma, \mathrm{E}$, and $u_{p}$ are, respectively, the end states of specific volume, pressure, energy, and particle velocity achieved from the passage of a shock wave. ${ }^{15-19} U_{s}$ is the shock front velocity relative to the initial material state of specific volume $\left(V_{0}\right)$, density $\left(\rho_{0}\right)$, pressure $\left(\sigma_{0}\right)$, particle velocity $\left(u_{0}\right)$, and energy $\left(\mathrm{E}_{0}\right)$.

The aim of the present work is to apply the isentropic stress reverberation approximation to the establishment of a relationship describing lower range of the equation of state using aluminum, structural steel, and polycarbonate. As for the principal Hugoniot, the relation being sought will be derived in terms of the material's particle velocity $\left(u_{p}\right)$ and the shock velocity $\left(U_{s}\right)$ of the material, in the form

$$
U_{s}=c_{b}+s u_{p}+Q u_{p}^{2},
$$

where $c_{b}$ is the sound velocity at near zero pressure corresponding to the initial equilibrium bulk compressibility of the medium, and the terms $s$ and $Q$ are fit parameters.

In Sec. II, the relations necessary to the proper interpretation of the experimental data are first derived, as previous uses of the method in literature have leapt to final results, circumventing the mention of intermediate steps. The requisite relations are presented in such a manner as to be directly applicable to any resulting set of experimental data. Next, the means of properly conducting the experiments are explained. Finally, the results are presented.

\section{THEORETICAL CONSIDERATIONS}

Consider the reverberations to equilibrium that occur as an incident shock wave interacts within a slender layer embedded in an otherwise homogenous structure. Figure 1 illustrates this scenario, where B.M. denotes the bulk material, and I.L., the inner layer. The mechanical responses of these materials are governed by the bulk material and inner layer Hugoniots, respectively, in the $\sigma-u_{p}$ plane. The impedance of the inner layer is specified as being small compared to that of the bulk material. In the time distance diagram, solid line characteristics represent compressive waves while dotted line characteristics represent unloading waves.

First, assume that a $\mathrm{C}+$ compressive wave, generated by a symmetrical impact, is propagating through the bulk material, yielding state 0 ' in the front plate. When this $\mathrm{C}+$ compressive pulse arrives at the inner layer, continuity at the interface between the bulk material and inner layer can only be achieved through intersection of their respective Hugoniots in the $\sigma-u_{p}$ plane. If the Hugoniot and unloading isentrope of the bulk material coincide, this assumption requires a C-tensile release wave of magnitude $\left(\sigma_{o^{\prime}}-\sigma_{1}\right)$ to be generated within the front plate of the bulk material and a $\mathrm{C}+$ compressive wave of magnitude $\sigma_{1}$ to be generated within the inner layer, resulting in state 1 . Likewise, when the $\mathrm{C}+$ compressive wave that was transmitted to the inner layer arrives at the back plate of the bulk material, continuity of the interface results in a $\mathrm{C}+$ compressive wave of magnitude $\sigma_{2}$, transmitted to the back plate and a C-compressive wave, of magnitude $\left(\sigma_{2}-\sigma_{1}\right)$, reflected within the inner layer, resulting in state 2 . As this process continues, the magnitude of stress within the inner layer will approach that of the initial incident pulse through multiple compressive reflections within the inner layer.

Introduction of in-situ stress gages onto both sides of the inner layer allows for the capture of temporal stress records associated with the reverberations to equilibrium. In the current case, these records will demonstrate the steplike loading profile associated with the process. Compressive steps observed on the front gage will directly relate to the stress at the odd numbered states 1, 3, and 5, while steps observed by the back gage will directly relate to the stress at the even numbered states 2, 4, and 6 . The Rankine-Hugoniot equations form a complete system when two state parameters are specified. There are two ways in which this requirement can be satisfied. First, the shock velocity within the inner layer could be obtained as a function of shock amplitude by comparison of the temporal records of the two gages and relating the time lag between the observed steps to the thickness of the inner layer. This method will be set aside in favor of the more rigorous method of impedance matching, where it will be required that the bulk material has a known Hugoniot.

Now, consider how the Hugoniot of the inner layer can be constructed from the known Hugoniot of the bulk plate material. In the $\sigma-u_{p}$ diagram depicted in Fig. 1(b), the reverberations associated with the back gage follow the principal Hugoniot of the bulk material, while those of the front gage follow the negatively sloped Hugoniot drawn from the impact velocity $\left(u_{i}\right)$. Assuming that the Hugoniot of the bulk material is well represented by Eq. (4), the RankineHugoniot conservation of momentum, Eq. (2), can be used to solve for the particle velocity associated with the measured stresses at each state. For the case of the back gage following the principal Hugoniot of the bulk material, the particle velocity of each successive step can be solved in terms of the measured stress in the form

$$
\sigma_{n}-\sigma_{n-2}=\rho_{n-2}\left(c_{b}+s u_{n}+Q u_{n}^{2}\right)\left(u_{n}-u_{n-2}\right),
$$

where the properties $\rho, c_{b}, s$, and $Q$ are taken from the bulk material's Hugoniot. In terms of Fig. 1(b), the subscript $n$ can be replaced with the even numbered states 2,4 , or 6 . The solution of Eq. (5) requires an iterative process where each additional jump in states (e.g., states 2-4, and 4-6) is solved in terms of the solution of Eq. (5) from the previous state jump. When solving for state 2 from state 0 , the initial density of the bulk material associated with state 0 is used. In contrast, 


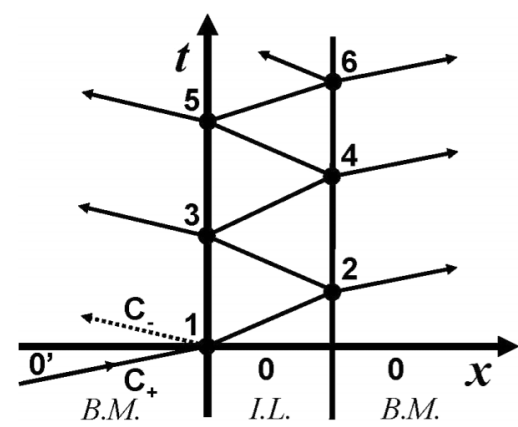

(a)

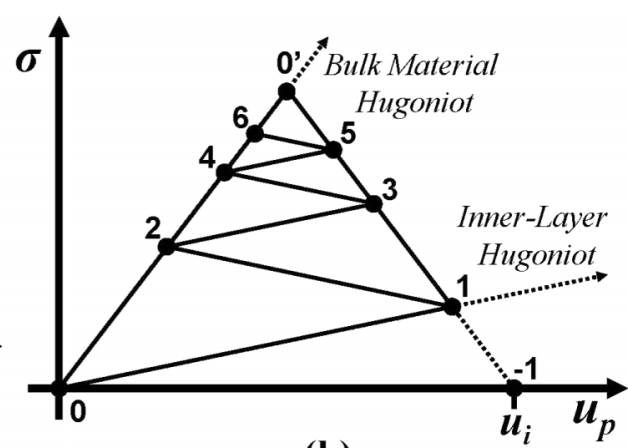

(b)

FIG. 1. Wave processes associated with an incident pulse acting on a thin layer in an otherwise homogenous structure where the inner layer (I.L.) is of low impedance with respect to the bulk material (B.M.); (a) $x-t$ diagram; (b) $u_{p}-\sigma$ plot.

the solution of state 4 from state 2 assumes that state 2 is the initial state in the jump equation and therefore requires the determination of the bulk material's density associated with state 2 . Likewise, any additional jump requires the solution of the density at the previous state, which will in turn represent the density of the defined initial state in Eq. (5). Through manipulation of the Rankine-Hugoniot conservation of mass, Eq. (1), the density at the even numbered states $(n=2,4,6)$ can be solved in terms of the density at the previous state in the form

$$
\rho_{n}=\rho_{n-2} \frac{c_{b}+s u_{n}+Q u_{n}^{2}}{\left(c_{b}+s u_{n}+Q u_{n}^{2}\right)-\left(u_{n}-u_{n-2}\right)},
$$

where each solution requires the particle velocity associated with the state to be generated from the preceding solution of Eq. (5).

The particle velocity associated with the odd numbered states $(n=1,3,5)$ can be solved in terms of the measured stress from the front gage in the form

$$
\begin{aligned}
\sigma_{n}-\sigma_{n-2}= & -\rho_{n-2}\left[\left(c_{b}+s\left(u_{i}-u_{n}\right)+Q\left(u_{i}-u_{n}\right)^{2}\right)\right] \\
& \times\left(u_{n}-u_{n-2}\right),
\end{aligned}
$$

where $u_{i}$ refers to the impact velocity and the density can be solved in the form

$\rho_{n}=\rho_{n-2} \frac{c_{b}+s\left(u_{i}-u_{n}\right)+Q\left(u_{i}-u_{n}\right)^{2}}{\left[c_{b}+s\left(u_{i}-u_{n}\right)+Q\left(u_{i}-u_{n}\right)^{2}\right]-\left(u_{n}-u_{n-2}\right)}$,

with the assumption that the density associated with state -1 , in Fig. 1(b), is the initial density $\left(\rho_{0}\right)$ of the bulk material. Eqs. (5)-(8) now allow for the determination of particle velocity given measured stress, which in turn permits the solution of any additional state parameters of interest with use of the Rankine-Hugoniot jump Eqs. (1)-(3). In order to construct the principal Hugoniot of the inner layer from an experiment, the particle velocities $\left(u_{n}\right)$ associated with the various states can be transformed to particle velocities along the principal Hugoniot of the inner layer $\left(u_{\text {principal }, n}\right)$ through use of the equation

$$
u_{\text {principal }, n}=\sum_{j=1}^{n-1}\left[(-1)^{j-1} 2 u_{j}\right]-(-1)^{n} u_{n}
$$

where $n$ is the associated state number.
Finally, the presence of radial release waves cannot be ignored, as they may potentially corrupt the experimental data. ${ }^{15-19}$ Fortunately, a simple geometric analysis provides a solution to this dilemma. Defining as $r$, the radial distance from the location of the gage to the edge of the specimen, and $D$, its distance from the impact face, then the time of arrival of the radial release waves can be defined as

$$
t_{r r}=\frac{\sqrt{D^{2}+r^{2}}}{c}
$$

where $c$ is an appropriate wave speed associated with the experiment.

\section{EXPERIMENTAL DESIGN}

\section{A. Materials studied}

Two sets of experiments, using either 6061 aluminum or A572 grade 50 structural steel as the bulk material and polycarbonate as the inner layer, were conducted to provide validation and confirm repeatability of the technique. By using two separate bulk materials, these experiments probed the method's ability to generate accurate Hugoniot states for the polycarbonate inner layer, independently of the specific bulk material properties. The design of these experiments necessitates the knowledge of values for the bulk wave speeds $\left(c_{b}\right)$ and initial densities $\left(\rho_{0}\right)$ of the materials. Bulk wave speeds can be calculated from measurements of longitudinal $\left(c_{l}\right)$ and shear $\left(c_{s}\right)$ wave speeds by using the well known relation

$$
c_{b}=\sqrt{c_{l}^{2}-\frac{4}{3} c_{s}^{2}}
$$

Longitudinal and shear wave speeds of the materials were measured using contact ultrasonic transducers. Densities were calculated from measurements of the volume and mass of the test samples. Table I contains a summary of these initial properties for these materials.

\section{B. Sample geometry}

\section{Aluminum/polycarbonate configuration}

Fig. 2 represents a time-distance diagram of the experiments that used 6061 aluminum as the bulk material. The aluminum impactor and test sample were both $45 \mathrm{~mm}$ in diameter. The thickness of the front face and impactor was 
TABLE I. Densities and wave speeds of the materials studied.

\begin{tabular}{lcccc}
\hline \hline Material & $\begin{array}{c}\rho_{0} \\
\left(\mathrm{Kg} / \mathrm{m}^{3}\right)\end{array}$ & $\begin{array}{c}c_{l} \\
(\mathrm{~m} / \mathrm{s})\end{array}$ & $\begin{array}{c}c_{s} \\
(\mathrm{~m} / \mathrm{s})\end{array}$ & $\begin{array}{c}c_{b} \\
(\mathrm{~m} / \mathrm{s})\end{array}$ \\
\hline 6061 aluminum & 2703 & 6420 & 3130 & 5310 \\
A572 grade 50 structural steel & 7814 & 5940 & 3260 & 4590 \\
Polycarbonate & 1193 & 2260 & 910 & 2000 \\
\hline \hline
\end{tabular}

$7.62 \mathrm{~mm}$. The thickness of the polycarbonate inner layer varied between $1.27 \mathrm{~mm}$ and $0.508 \mathrm{~mm}$, where tests 1 , 2,3 , and 4 used polycarbonate thicknesses of $1.27 \mathrm{~mm}$, $0.838 \mathrm{~mm}, 0.508 \mathrm{~mm}$, and $0.508 \mathrm{~mm}$, respectively. In all cases, stress gages were embedded between the two interfaces of the polycarbonate inner layer and the bulk material. The aluminum back face of the sample was chosen to be $12.7 \mathrm{~mm}$ to ensure that no reflections from its free surface would reach the stress gage locations during the measurement period of the experiments.

Within Fig. 2, compressive waves and release waves are, respectively, drawn with solid and dotted characteristic lines. The radial release wave, indicative of the violation of one-dimensional strain, was constructed using Eq. (10). The chosen impactor thickness induces a loading duration of $2.9 \mu$ s. Fig. 2 clearly highlights the complications associated with radial release waves with samples having relatively small diameter to thickness ratios. Observing the respective arrival times of the $\mathrm{C}+$ unloading and the radial release to the polycarbonate inner layer, the chosen thickness of the impactor is shown to maximize the ring-up period achievable with $45 \mathrm{~mm}$ diameter samples.

Because of the different thicknesses of the polycarbonate inner layers used in the four tests, the stress reflections within the inner layer are not depicted in Fig. 2. Using the bulk wave speed of $2000 \mathrm{~m} / \mathrm{s}$ for polycarbonate, the layer thickness for test 1 will result in compressive reflections in the inner layer every $0.635 \mu \mathrm{s}$. Therefore, jumps in the stress observed at each interface will initially occur every $1.27 \mu$ s. The

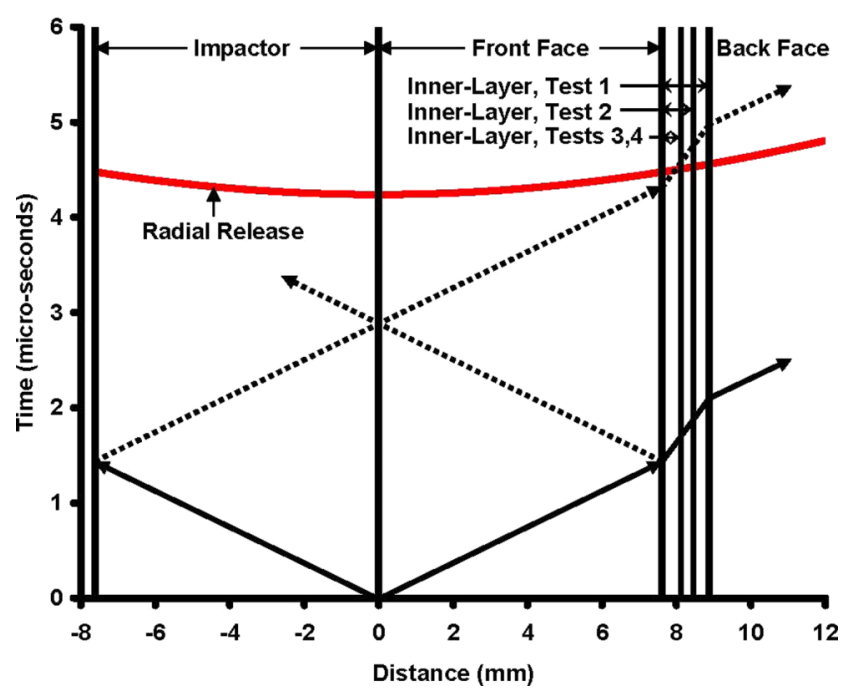

FIG. 2. Time-distance diagram for the isentropic experiments using 6061 aluminum as the bulk material and polycarbonate as the inner layer. shock velocity within the polycarbonate layer will increase for each subsequent reflection as a result of the increasing pressure within. Thus, the time lag between stress jumps will decrease with increasing state numbers. In order to address this condition, the first polycarbonate thickness was chosen to ensure that these stress jumps would be sufficiently long to accurately resolve the states from the stress records. For test 2 , the polycarbonate layer thickness was chosen to produce compressive reflections every $0.42 \mu \mathrm{s}$, resulting in initial time lags of $0.84 \mu$ s between stress jumps at each interface. Likewise, the polycarbonate layer thickness chosen for tests 3 and 4 produce compressive reflections every $0.25 \mu \mathrm{s}$, and therefore, lag times between stress jumps at each interface will initially occur every $0.50 \mu \mathrm{s}$.

\section{Steel/polycarbonate configuration}

Fig. 3 displays a time-distance diagram of the experiments that used A572 grade 50 structural steel as the bulk material. Similar to the specimens in Sec. III B 1, the steel impactor and test sample were both $45 \mathrm{~mm}$ in diameter. The thickness of the front face and impactor was $5.08 \mathrm{~mm}$. The thickness of the polycarbonate inner layer was $0.508 \mathrm{~mm}$. Stress gages were embedded between the two interfaces of the polycarbonate inner layer and the bulk material. The steel back face of the sample was chosen to be $7.62 \mathrm{~mm}$ to ensure that no reflections from its free surface would reach the stress gage locations during the measurement period of the experiments.

The chosen impactor thickness induces a loading duration of $2.2 \mu \mathrm{s}$. In this case, the arrival of the radial release to the polycarbonate inner layer occurs much later in time than the arrival of the $\mathrm{C}+$ unloading wave. Thus, the impactor thickness could have been increased to maximize the ring-up period within the polycarbonate. However, the resulting increase in impactor weight would then have become an impediment to achieving higher impact speeds. The time-distance diagram indicates that five states can be captured at each interface. The compressive reflections in the inner layer occur every $0.25 \mu \mathrm{s}$.

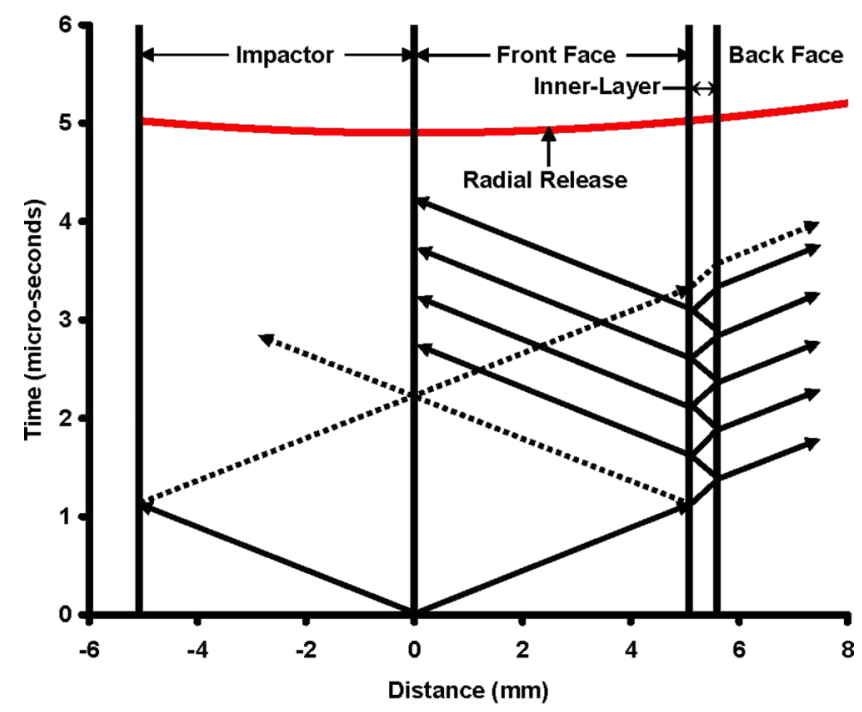

FIG. 3. Time-distance diagram for the isentropic experiments using steel as the bulk material and polycarbonate as the inner layer. 
Therefore, jumps in the stress observed at each interface will initially occur every $0.50 \mu \mathrm{s}$.

\section{Plate impact experiments}

Plate impact experiments were conducted in a vacuum chamber with the use of a $50 \mathrm{~mm}$ bore single stage helium driven gas gun. Polyvinyl chloride (PVC) sabots were used to carry the impactors down the $2 \mathrm{~m}$ long gun barrel while specimens were supported, awaiting impact, by means of small PVC rings that were weakly bonded to a metal sample holder. In order to attain acceptable impact planarity, the sabots were designed such as to impact the specimen before fully exited the barrel of the gun. Flyer velocities were captured by means of two separate through-beam systems, each consisting of two light sources and two receivers. Velocity was inferred by dividing the distance between the receivers by the time between interruptions. Stress profiles were captured with the use of $50 \Omega$ manganin stress gages manufactured by Dynasen. ${ }^{20}$

\section{Hugoniot parameters}

Hugoniot parameters for the materials used were obtained from published data for 6061 aluminum and polycarbonate found in work performed at LANL. ${ }^{2}$ For aluminum, the relationship is well represented by the linear form of Eq. (4)

$$
U_{s}=5350+1.34 u_{p} .
$$

Within the range of particle velocities from 0 to $1000 \mathrm{~m} / \mathrm{s}$, for polycarbonate, a linear relation suffices

$$
U_{s}=2000+2.122 u_{p}
$$

If the range of particle velocities is extended to $4000 \mathrm{~m} / \mathrm{s}$, a polynomial fit is required

$$
U_{s}=2000+2.1612 u_{p}-0.0002 u_{p}^{2} .
$$

Substituting either Eqs. (13) or (14) in Eq. (2), with an initial density $\left(\rho_{0}\right)$ of $1193 \mathrm{Kg} / \mathrm{m} 3$, yields the principal Hugoniot of polycarbonate in $\sigma-u_{p}$ space.

Although there are numerous Hugoniots for steel published in the literature, none has been derived for A572 grade 50 structural steel. Nevertheless, Visser et al. ${ }^{21}$ have recently tested this specific steel over a moderate range of impact velocities. Within this investigation, five symmetrical plate impacts were executed, and stress histories were captured with the use of manganin gages. Even though the goal of their research was not intended to generate the Hugoniot of the material, these experiments possessed an excellent configuration to obtain Hugoniot states through traditional analyses. Since the impact conditions used the same material impactor as the test sample, symmetry of the Hugoniots would result in particle velocities that are exactly half the impact velocities. Additionally, since the stress gages were embedded within the steel targets, the experimental stress records would capture an adequate representation of the pressures associated with these particle velocities. A summary of the five tests is reproduced in Table II, along with associated shock velocities calculated using of Eq. (2), with an initial density of $7814 \mathrm{Kg} / \mathrm{m}^{3}$.
TABLE II. Hugoniot states for A572 grade 50 structural steel.

\begin{tabular}{lllc}
\hline \hline$u_{i}(\mathrm{~m} / \mathrm{s})$ & $\sigma(\mathrm{GPa})$ & $u_{p}(\mathrm{~m} / \mathrm{s})$ & $U_{s}(\mathrm{~m} / \mathrm{s})$ \\
\hline 241 & 4.2 & 120.5 & 4461 \\
382 & 7 & 191 & 4690 \\
405 & 7.33 & 202.5 & 4632 \\
418 & 7.52 & 209 & 4605 \\
477 & 8.82 & 238.5 & 4733 \\
\hline \hline
\end{tabular}

Reference 22 specifies a linear relationship for 4340 steel, a material similar to the one of interest in the present case,

$$
U_{s}=4578+1.33 u_{p} .
$$

Transposing this relation into Eqs. (15) and (2), its translation into the $\sigma-u_{p}$ space is compared with the experimentally determined states for A572 grade 50 structural steel.

There is an excellent agreement between the Hugoniot of 4340 steel and the experimentally determined Hugoniot states for A572 grade 50 structural steel within the range of the experimental data. It is possible that the two data sets might deviate from each other as stress and particle velocities increase. However, since the present experiments will not exceed the indicated range, the fit parameters from Eq. (15) may safely be appropriated (Fig. 4).

\section{RESULTS}

In the experiments that used aluminum as the bulk material, three distinct steps were observed by each of the two stress gages, yielding six states per test. Ten states were successfully captured in the experiments that used steel as the bulk material. These experiments demonstrated that the thickness of the polycarbonate inner layer influences the resolution of each stress plateau. The stress records for the $1.27 \mathrm{~mm}$ polycarbonate inner layer used in test 1 of the aluminum/ polycarbonate configuration provided relatively long time durations at each stress jump, where clear plateaus facilitated

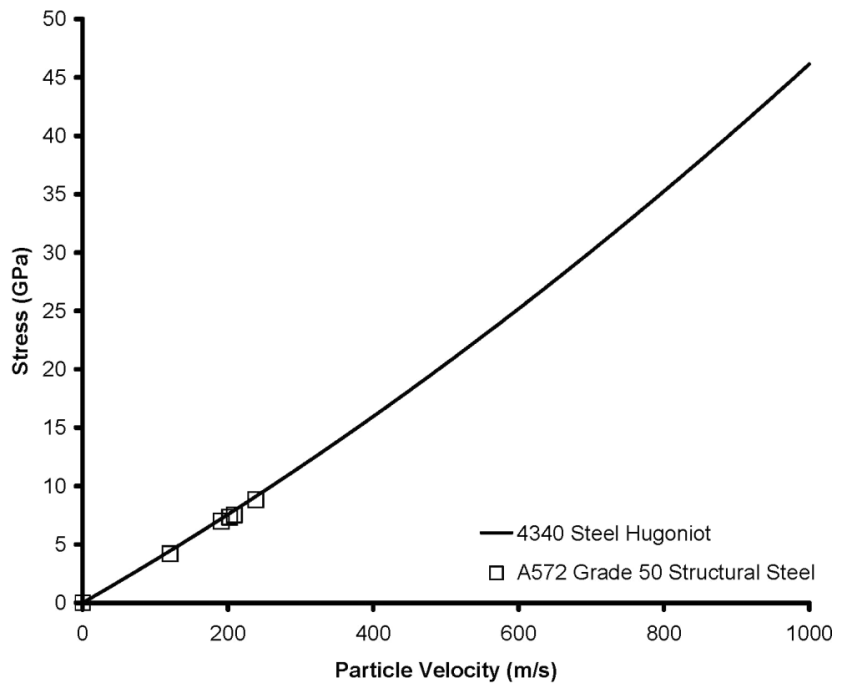

FIG. 4. Hugoniot of steel in stress-particle velocity space. 


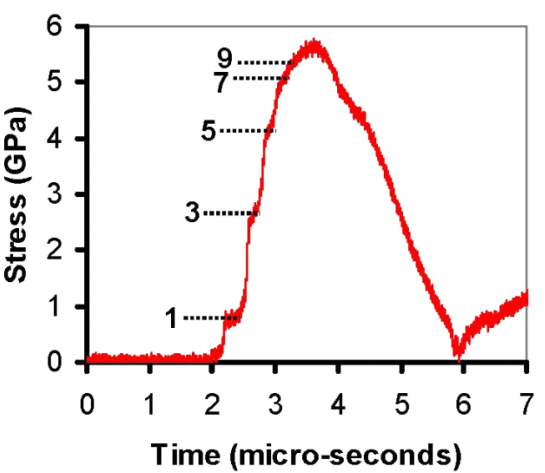

(a)

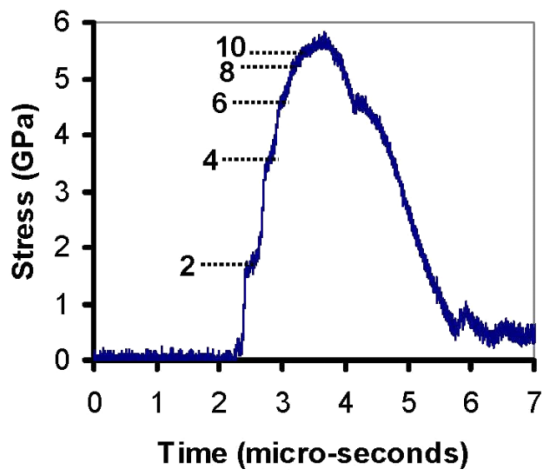

(b)

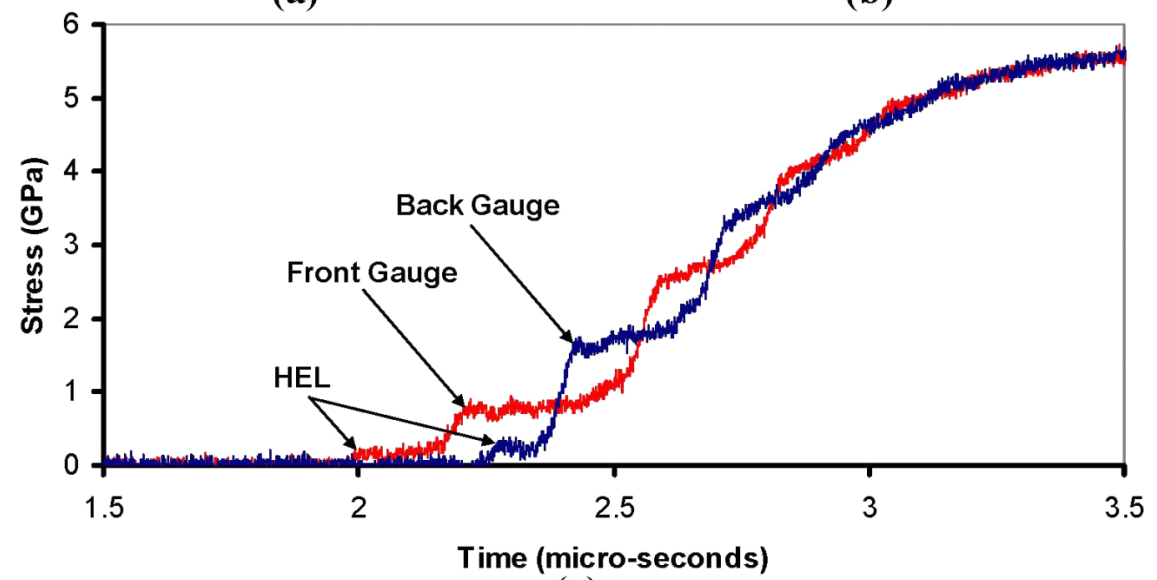

(c)

FIG. 5. Stress records from the first test on steel/polycarbonate with an inner layer thickness of $0.508 \mathrm{~mm}$ and an impact velocity of $300 \mathrm{~m} / \mathrm{s}$; (a) front gage; (b) back gage; (c) coupled plot of both gages.

the identification of the stress magnitude at each state. In contrast, the stress records for the $0.508 \mathrm{~mm}$ polycarbonate inner layer possessed much shorter time durations at each stress jump, often resulting in no clear plateau. In these cases, the stress at each state was estimated by taking the midpoint between the partially sloping signals of each stress plateau. A history of the stress evolution within the polycarbonate inner layer for test 1 of the steel/polycarbonate configuration can be found in Fig. 5. Parts (a) and (b) contain the stress records from the front and back gages, respectively. Part (c) present a coupled stress records of the front and back gages overlaid to produce a single plot. The various stresses at states are clearly labeled. Likewise, the HEL of the steel is easily noted in the initial loading profile as indicated in Fig. 5(c).

Tables III and IV contain the results for experiments conducted with the aluminum/polycarbonate and steel/polycarbonate configurations, respectively. Within these tables, the test number, inner layer thickness $\left(T_{\text {I.L. }}\right)$, impact velocity $\left(u_{i}\right)$, state number, stress $(\sigma)$, and particle velocity $\left(u_{p}\right)$ determined through solution of Eqs. (5)-(8) can be found. In order to construct the principal Hugoniot of the polycarbonate inner layer from the calculated particle velocities at each state, Eq. (9) was used. These results are tabulated in the column marked $u_{\text {principal }}$. Additionally, the shock velocities $\left(U_{s}\right)$ in the polycarbonate inner layer at each state were calculated with use of the Rankine-Hugoniot conservation of momentum Eq. (2), where the principal particle velocities and density of polycarbonate were used.
To further confirm the methodology, Fig. 6 was generated to compare the experimentally derived $\sigma-u_{p}$ data sets to published data. The published data are represented by closed diamonds. The states obtained from the aluminum/polycarbonate tests $1,2,3$, and 4 are depicted by open squares, triangles, circles, and diamonds, respectively. The states obtained from the steel/polycarbonate tests 1 and 2 are, respectively, depicted

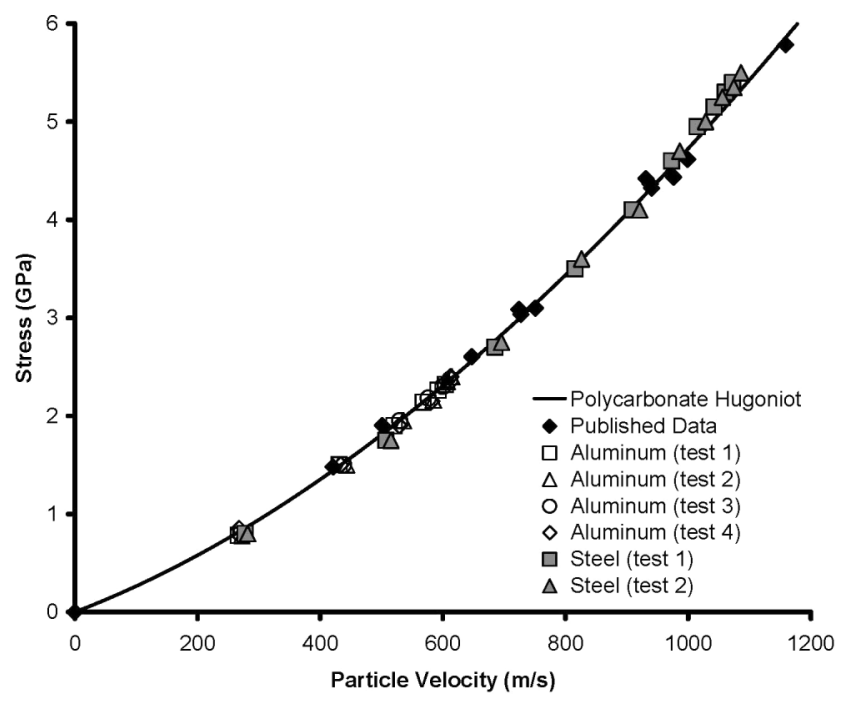

FIG. 6. Stress-particle velocity plot of polycarbonate's Hugoniot comparing the shocked states obtained through the use of the HRUM to published data. 
TABLE III. Hugoniot states of polycarbonate determined using aluminum as the bulk material.

\begin{tabular}{|c|c|c|c|c|c|c|c|}
\hline $\begin{array}{l}\text { Test } \\
\text { No. }\end{array}$ & $T_{I . L .}(\mathrm{mm})$ & $u_{i}(\mathrm{~m} / \mathrm{s})$ & $\begin{array}{c}\text { State } \\
\text { No. }\end{array}$ & $\sigma(\mathrm{GPa})$ & $u_{p}(\mathrm{~m} / \mathrm{s})$ & $u_{\text {principal }}(\mathrm{m} / \mathrm{s})$ & $U_{s}(\mathrm{~m} / \mathrm{s})$ \\
\hline \multirow{6}{*}{1} & \multirow{6}{*}{1.27} & \multirow{6}{*}{320} & 1 & 0.78 & 266 & 266 & 2486 \\
\hline & & & 2 & 1.5 & 102 & 431 & 2955 \\
\hline & & & 3 & 1.9 & 192 & 521 & 3098 \\
\hline & & & 4 & 2.14 & 144 & 568 & 3197 \\
\hline & & & 5 & 2.26 & 168 & 592 & 3239 \\
\hline & & & 6 & 2.32 & 156 & 604 & 3258 \\
\hline \multirow{6}{*}{2} & \multirow{6}{*}{0.838} & \multirow{6}{*}{326} & 1 & 0.78 & 272 & 272 & 2431 \\
\hline & & & 2 & 1.5 & 102 & 443 & 2875 \\
\hline & & & 3 & 1.95 & 194 & 535 & 3092 \\
\hline & & & 4 & 2.16 & 145 & 584 & 3138 \\
\hline & & & 5 & 2.35 & 168 & 607 & 3285 \\
\hline & & & 6 & 2.4 & 161 & 614 & 3316 \\
\hline \multirow{6}{*}{3} & \multirow{6}{*}{0.508} & \multirow{6}{*}{325} & 1 & 0.8 & 270 & 270 & 2515 \\
\hline & & & 2 & 1.5 & 102 & 438 & 2906 \\
\hline & & & 3 & 1.95 & 193 & 530 & 3125 \\
\hline & & & 4 & 2.18 & 147 & 576 & 3211 \\
\hline & & & 5 & 2.3 & 170 & 600 & 3253 \\
\hline & & & 6 & 2.37 & 159 & 612 & 3290 \\
\hline \multirow{6}{*}{4} & \multirow{6}{*}{0.508} & \multirow{6}{*}{326} & 1 & 0.85 & 268 & 268 & 2696 \\
\hline & & & 2 & 1.5 & 102 & 433 & 2938 \\
\hline & & & 3 & 1.9 & 198 & 529 & 3048 \\
\hline & & & 4 & 2.15 & 145 & 582 & 3135 \\
\hline & & & 5 & 2.35 & 168 & 606 & 3294 \\
\hline & & & 6 & 2.4 & 161 & 613 & 3325 \\
\hline
\end{tabular}

by shaded squares and triangles. The solid line in Fig. 6 depicts the Hugoniot curve for polycarbonate, constructed with use of Eq. (2), while using the $U_{s}-u_{p}$ relationship defined by Eq. (14). The agreement between the experimentally obtained states and the Hugoniot curve of polycarbonate clearly demonstrates that the method successfully generates states along the Hugoniot of an inner layer embedded within a well classified bulk material. The error between the published

TABLE IV. Hugoniot states of polycarbonate determined using steel as the bulk material.

\begin{tabular}{|c|c|c|c|c|c|c|c|}
\hline \multirow{2}{*}{$\begin{array}{l}\text { Test } \\
\text { No. }\end{array}$} & \multirow[b]{2}{*}{$T_{I . L .}(\mathrm{mm})$} & \multirow[b]{2}{*}{$u_{i}(\mathrm{~m} / \mathrm{s})$} & \multirow{2}{*}{$\begin{array}{l}\text { State } \\
\text { No. }\end{array}$} & \multirow[b]{2}{*}{$\sigma(\mathrm{GPa})$} & \multirow[b]{2}{*}{$u_{p}(\mathrm{~m} / \mathrm{s})$} & \multirow[b]{2}{*}{$u_{\text {principal }}(\mathrm{m} / \mathrm{s})$} & \multirow[b]{2}{*}{$U_{s}(\mathrm{~m} / \mathrm{s})$} \\
\hline & & & & & & & \\
\hline \multirow{10}{*}{1} & \multirow{10}{*}{0.508} & \multirow{10}{*}{300} & 1 & 0.8 & 278 & 278 & 2445 \\
\hline & & & 2 & 1.75 & 48 & 507 & 2929 \\
\hline & & & 3 & 2.7 & 266 & 685 & 3346 \\
\hline & & & 4 & 3.5 & 95 & 816 & 3643 \\
\hline & & & 5 & 4.1 & 189 & 909 & 3829 \\
\hline & & & 6 & 4.6 & 124 & 973 & 4013 \\
\hline & & & 7 & 4.95 & 166 & 1015 & 4140 \\
\hline & & & 8 & 5.15 & 139 & 1042 & 4194 \\
\hline & & & 9 & 5.3 & 157 & 1061 & 4242 \\
\hline & & & 10 & 5.4 & 145 & 1072 & 4274 \\
\hline \multirow{10}{*}{4} & \multirow{10}{*}{0.508} & \multirow{10}{*}{304} & 1 & 0.8 & 282 & 282 & 2410 \\
\hline & & & 2 & 1.75 & 48 & 515 & 2883 \\
\hline & & & 3 & 2.75 & 229 & 696 & 3356 \\
\hline & & & 4 & 3.6 & 98 & 826 & 3699 \\
\hline & & & 5 & 4.1 & 193 & 921 & 3780 \\
\hline & & & 6 & 4.7 & 127 & 986 & 4045 \\
\hline & & & 7 & 5 & 169 & 1028 & 4127 \\
\hline & & & 8 & 5.25 & 141 & 1056 & 4221 \\
\hline & & & 9 & 5.35 & 160 & 1074 & 4228 \\
\hline & & & 10 & 5.5 & 148 & 1086 & 4298 \\
\hline
\end{tabular}


data and the Hugoniot curve of polycarbonate is of the same order as that of the data obtained through the current method. The published data were obtained through carefully controlled single shock experiments, whereas the data sets in the Hugoniot ring-up method (HRUM) were obtained through re-shocking of the inner layer. The agreement between the states obtained through traditional methods to those obtained using the HRUM demonstrates that materials follow the same path (Hugoniot) through stress-particle velocity space in either cases of single shock or re-shock events for very mild shock states.

Referring back to Fig. 6, it is notable to draw attention to the peak pressures that were achieved within the polycarbonate inner layer.

The nature of the ring-up mechanism is such that peak pressures of $2.4 \mathrm{GPa}$ and $5.5 \mathrm{GPa}$ were excited by the aluminum/polycarbonate and steel/polycarbonate configurations, respectively. Had conventional single shock experiments been used to determine these upper data sets, researchers would traditionally choose an impactor material with a high hydrodynamic impedance. For example, consider the impact velocity that would be required to excite these peak pressures through a single shock experiment using tungsten carbide (higher impedance than both aluminum and steel) as the impactor material. Using published Hugoniot of tungsten carbide, ${ }^{2}$ it is easily be shown that the impact velocity would have to be, respectively, over $640 \mathrm{~m} / \mathrm{s}$ and $1150 \mathrm{~m} / \mathrm{s}$ to achieve pressures of $2.4 \mathrm{GPa}$ and $5.5 \mathrm{GPa}$ within a polycarbonate test sample. Considering that most plate impact apparatuses are limited to some upper end impact velocity, reloading proves advantageous in accessing regimes that would not have been attainable through traditional single shock experiments.

\section{CONCLUDING REMARKS}

Isentropic stress reverberations were used to induce shock reflections in a low impedance inner layer embedded within high impedance bulk structures. By capturing temporal records of the stress evolution on either side of the inner layer, step-like loading profiles were obtained that allowed for the capture of multiple Hugoniot states. The mathematical framework used in this technique used the classical RankineHugoniot equations in the method of impedance matching, where the bulk material was required to have a known Hugoniot. Validation of the technique was achieved by testing well classified materials in order to facilitate comparison of the Hugoniots generated from the method with published data from literature. The technique was shown to accurately generate states along the Hugoniot of the inner layer, where the number of states acquired is directly linked to the experimental design. Experiments using 6061 aluminum and polycarbonate as the bulk material and inner layer, respectively, generated six Hugoniot states for the polycarbonate. Experiments using A572 grade 50 structural steel as the bulk material generated ten Hugoniot states for the polycarbonate. In these experiments, it has been shown that an additional advantage of the method over conventional single shock experiments is the elevated peak pressure that can be achieved within the inner layer for any given impact velocity. The method is limited to regimes where Hugoniot and isentrope coincide, as was the case for the current investigations. At extreme pressures, applications of the method would break down, since behavior at those levels can no longer be considered isentropic.

${ }^{1}$ S. Sikka, Bull. Mater. Sci. 15, 35 (1992).

${ }^{2}$ S. P. Marsh, LASL Shock Hugoniot Data (University of California Press, 1980), Vol. 5.

${ }^{3}$ K. Cochrane, M. Desjarlais, T. Haill, J. Lawrence, M. Knudson, and G. Dunham, Sandia Report No. SAND2006-1739, 2006, http://www.sandia. gov/pulsedpower/prog_cap/pub_papers/061739.pdf.

${ }^{4}$ Z. Rosenberg and Y. Yeshurun, J. Appl. Phys. 58, 3077 (1985).

${ }^{5}$ Z. Rosenberg, Y. Meybar, and D. Yaziv, J. Phys. D: Appl. Phys. 14, 261 (1981).

${ }^{6}$ G. Gathers, J. Appl. Phys. 67, 4090 (1990).

${ }^{7}$ D. Chen, Y. Yu, Z. Yin, H. Wang, and G. Liu, Int. J. Impact Eng. 31, 811 (2005).

${ }^{8}$ G. Willmott and W. Proud, Int. J. Rock Mech. Min. Sci. 44, 228 (2007).

${ }^{9}$ Y. Berlinsky and Z. Rosenberg, J. Phys. D: Appl. Phys. 13, 861 (1980).

${ }^{10}$ Y. Sato, M. Komori, and W. Kikuchi, J. Mater. Process. Technol. 85, 43 (1999).

${ }^{11}$ P. Lysne, R. Boade, C. Percival, and O. Jones, J. Appl. Phys. 40, 3786 (1969).

${ }^{12}$ Z. Rosenberg, J. Appl. Phys. 52, 4000 (1981).

${ }^{13}$ D. Reisman, A. Toor, R. Cauble, C. Hall, J. Asay, M. Knudson, and M. Furnish, J. Appl. Phys. 89, 1625 (2001).

${ }^{14}$ J. B. Aidun and Y. Gupta, J. Appl. Phys. 69, 6998 (1991).

${ }^{15}$ M. Meyers, Dynamic Behavior of Materials (John Wiley \& Sons, 1994).

${ }^{16}$ D. Grady, L. Davison, and M. Shahinpoor, High-Pressure Shock Compression of Solids II (Springer, 1996).

${ }^{17}$ T. Antoun, D. Curran, S. Razorenov, L. Seaman, G. Kanel, and A. V. Utkin, Spall Fracture (Springer, 2003).

${ }^{18}$ W. Anderson, F. Cherne, B. Glushak, M. Zhernokletov, and M. Zocher, Material Properties Under Intensive Dynamic Loading (Springer, 2006).

${ }^{19} \mathrm{G}$. I. Kanel and V. E. Fortov, Shock-Wave Phenomena and the Properties of Condensed Matter (Springer, 2004).

${ }^{20}$ Dynasen, Piezoresistive Pulse Power Supply Model CK2-50/0.050-300 Instruction Manual, Dynasen, Inc., Goleta, CA, 1996.

${ }^{21}$ W. Visser, Y. Sun, O. Gregory, G. Plume, C.-E. Rousseau, and H. Ghonem, Mater. Sci. Eng.: A 528, 7857 (2011).

${ }^{22}$ D. Steinberg, Report No. UCRL-MA-106439, 1991-1996. 